\title{
Ventricular tachycardia sensitive to antitachycardia pacing in a patient with severely symptomatic Brugada syndrome
}

\author{
Takashi Nakashima $^{1}$ (D) Nobuhiro Takasugi ${ }^{1} \cdot$ Yuki Sahashi $^{1} \cdot$ Keita Suzuki $^{2} \cdot$ Tomoki Kubota $^{3} \cdot$ Takuma Aoyama $^{2}$. \\ Masanori Kawasaki ${ }^{1}$
}

Received: 26 August 2018 / Accepted: 3 October 2018 / Published online: 26 October 2018

(C) Springer Science+Business Media, LLC, part of Springer Nature 2018

\begin{abstract}
A 36-year-old man was resuscitated after cardiopulmonary arrest due to ventricular fibrillation (VF). He had no family history of sudden cardiac death. Echocardiography did not reveal any cardiac abnormalities. An ECG showed a spontaneous type 1 Brugada (BrS) ECG pattern in leads V1 and V2 (Fig. 1a). A splice cite variation in SCN5A, c.4810+4G>A, was identified in the genetic analysis. Thus, he was diagnosed with $\mathrm{BrS}$ and an implantable cardioverter-defibrillator (ICD) was implanted. During 1 year after implantation of ICD, he received 18 appropriate ICD shocks for VF (Fig. 1b). After administration of oral quinidine $1200 \mathrm{mg}$ /day and cilostazol $200 \mathrm{mg}$ /day therapy, no appropriate ICD shocks were delivered for 2 years. However, a
\end{abstract}

subsequent episode of a relatively regular ventricular tachycardia (VT), which later converted into fast VT, was documented and successfully terminated by antitachycardia pacing (ATP) (Fig. 1c).

One potential mechanism is that drug administration modified the arrhythmogenic substrate and thus facilitated the conversion of malignant VT into a more stable and regular form of VT sensitive to ATP.

Although some cases have described VT sensitive to ATP in patients with $\mathrm{BrS}$, the present case is unique in that $\mathrm{VT}$ sensitive to ATP was observed 2 years after drug administration in a severely symptomatic BrS patient, who had several appropriate ICD shocks for VF.

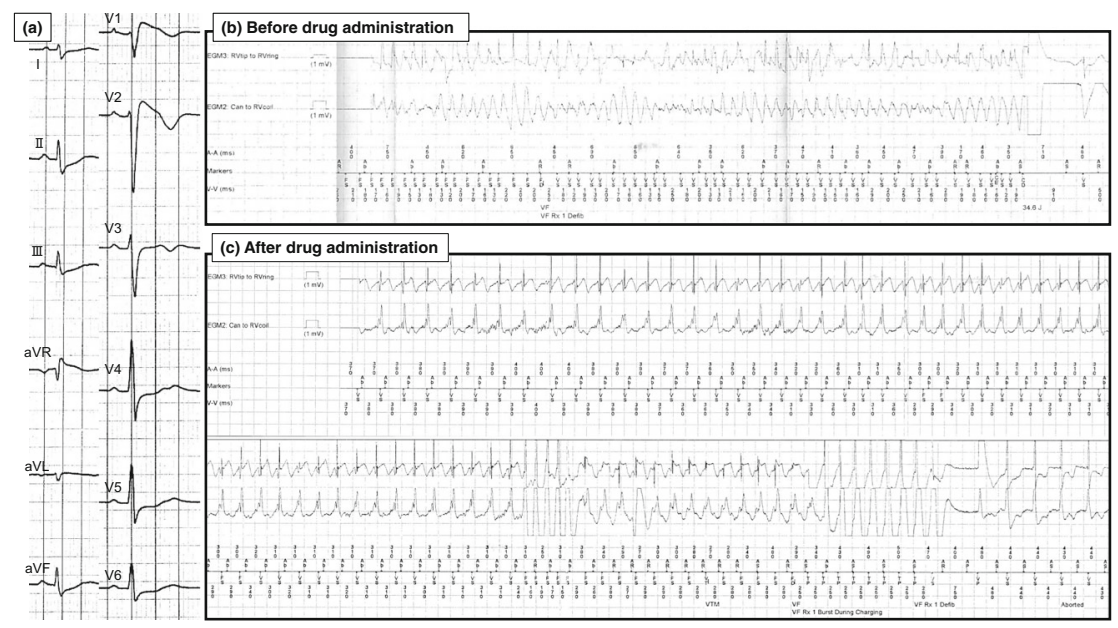

Fig. 1 ECG patterns (a), before drug administration (b), and after drug administration (c)

Takashi Nakashima

takashin727jw@yahoo.co.jp

1 Department of Cardiology, Graduate School of Medicine, Gifu University, Gifu, Japan

2 Department of Cardiology, Kizawa Memorial Hospital, Minokamo, Japan

3 Department of Cardiology, Gifu Municipal Hospital, Gifu, Japan 